Provided for non-commercial research and education use. Not for reproduction, distribution or commercial use.

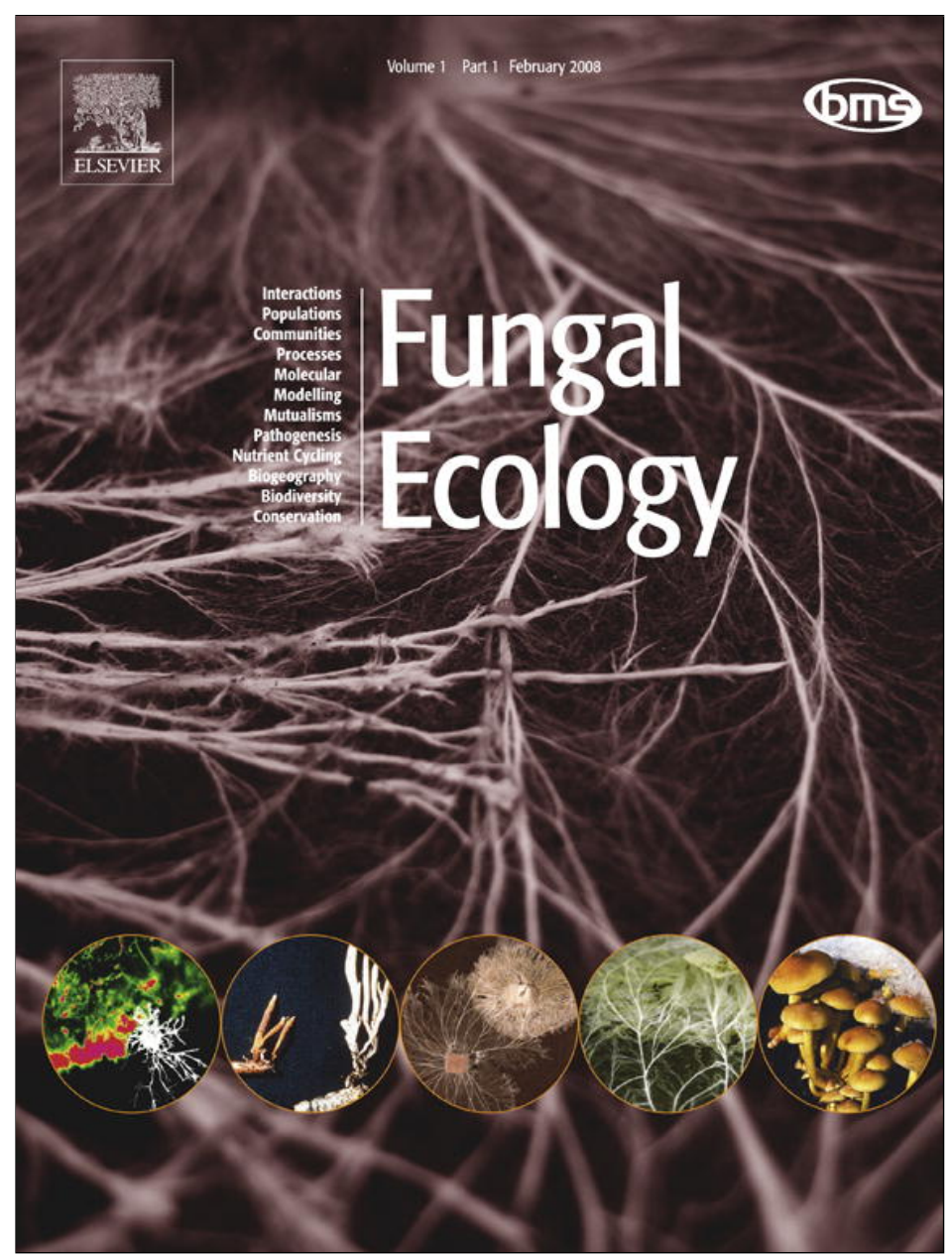

This article appeared in a journal published by Elsevier. The attached copy is furnished to the author for internal non-commercial research and education use, including for instruction at the authors institution and sharing with colleagues.

Other uses, including reproduction and distribution, or selling or licensing copies, or posting to personal, institutional or third party websites are prohibited.

In most cases authors are permitted to post their version of the article (e.g. in Word or Tex form) to their personal website or institutional repository. Authors requiring further information regarding Elsevier's archiving and manuscript policies are encouraged to visit:

http://www.elsevier.com/copyright 


\title{
Environmental detection of Penicillium marneffei and growth in soil microcosms in competition with Talaromyces stipitatus
}

\author{
Elizabeth PRYCE-MILLER ${ }^{1}$, David AANENSEN ${ }^{a}$, Nongnuch VANITTANAKOM $^{b}$, \\ Matthew C. FISHER ${ }^{a, *}$ \\ ${ }^{a}$ Department of Infectious Disease Epidemiology, Imperial College Faculty of Medicine, London, United Kingdom \\ ${ }^{b}$ Department of Microbiology, Microbiology Unit, Central laboratory, Faculty of Medicine, Chiang Mai University, Thailand
}

\section{A R T I C L E I N F O}

Article history:

Received 5 December 2007

Accepted 14 February 2008

Published online 18 April 2008

This manuscript is dedicated to the memory of Dr Pryce-Miller, 7th November 1976 to 11th September 2007.

Keywords:

Endemic

Environmental

Fungus

Mycosis

Penicillium

Quantitative environmental PCR

\begin{abstract}
A B S T R A C T
Penicillium marneffei is an endemic mycosis of humans in southeast Asia. Epidemiological data have shown that exposure to soil and season increase the risk of infection, and it is assumed that the main environmental reservoir is in soil. We sampled soils from a P. marneffei - endemic region of Thailand and confirmed by quantitative PCR and sequencing that P. marneffei DNA can be detected, a finding that we replicated over three sampling seasons. P. marneffei-positive and -negative sampling locations can be viewed using a dynamic browser located at www.spatialepidemiology.net/pmarneffei. We subsequently examined the hypothesis that P. marneffei isolates representing the two major phylogeographic clades of this species can grow in: (i) soil and (ii) competition against the closely related species, Talaromyces stipitatus, in a model soil environment. P. marneffei was not detected in nonsterile soil microcosms $14 \mathrm{~d}$ post inoculation, showing that the pathogen is unable to compete against complete soil fauna under our laboratory conditions. However, both isolates of P. marneffei persisted and increased in biomass when inoculated into sterile soil. P. marneffei stably co-existed with T. stipitatus, and that the main competitive interaction was the inhibition of T. stipitatus growth at low spore application by the 'Eastern' isolate of P. marneffei. We conclude that P. marneffei is present in soils within endemic regions, and is able to grow in soil under certain conditions. More research is required to ascertain the specific conditions that regulate the growth of P. marneffei in soils in natural environments.
\end{abstract}

(c) 2008 Elsevier Ltd and The British Mycological Society. All rights reserved.

\section{Introduction}

Penicillium marneffei is a mitosporic (asexual) pathogenic fungus of the Trichocomaceae family, and is endemic to southeast Asia (Ajello et al. 1995). P. marneffei has emerged as a significant human mycosis since the 1980's, paralleling the increasing incidence of the Human Immunodeficiency Virus
(HIV) within this region. The genus Penicillium contains over 200 species, many of which have a world-wide distribution in soil or decaying vegetation (Pitt 1988). P. marneffei is, however, unusual for several reasons: (i) the fungus is highly endemic, only being found across a narrow band of tropical southeast Asia (Supparatpinyo et al. 1994); (ii) it is the only member of the genus that exhibits temperature-dependent

\footnotetext{
* Corresponding author. Department of Infectious Disease Epidemiology, Imperial College Faculty of Medicine, St. Mary's Campus, Norfolk Place, London W2 1PG, United Kingdom. Tel.: +44 207594 3801; fax: +44 2072628140.

E-mail address: matthew.fisher@imperial.ac.uk (M.C. Fisher).

URL: http://www1.imperial.ac.uk/medicine/people/matthew.fisher

${ }^{1}$ Deceased author.

1754-5048/\$ - see front matter @ 2008 Elsevier Ltd and The British Mycological Society. All rights reserved.

doi:10.1016/j.funeco.2008.02.002
} 
dimorphic growth as an intracellular fission yeast; and (iii) it is the only member of the genus that can behave as a primary animal pathogen.

Whilst many studies have focused upon defining the ecotypes where certain Penicillium species dominate (Grishkan et al. 2003; Johansson 2001; Kjøller \& Struwe 1982; Paul \& Clarke 1970), similar studies have not been carried out for P. marneffei. It has been shown that a history of exposure to soil, especially in the rainy season, increases the risk of infection with P. marneffei (Chariyalertsak et al. 1997). It has only been cultivated once from a soil sample (Chariyalertsak et al. 1996), it is slow-growing, and this low rate of recovery may be due to the lack of a specific, selective, medium. The fungus has been found to naturally infect a high proportion of burrowing bamboo rats, an animal common to the region (Capponi et al. 1956; Chariyalertsak et al. 1996; Deng et al. 1986). However, it is not yet clear whether these bamboo rat infections represent an obligate stage in the P. marneffei life cycle. P. marneffei isolates with identical multilocus genotypes are shared between humans and bamboo rat species in Thailand and India (Fisher et al. 2005), showing bamboo rats are a possible zoonotic source for human infections (Fisher et al. 2004b). On the other hand, it is equally possible that bamboo rats and humans have become infected from a common environmental source. Determining the ability of this fungus to survive and compete in the natural environment is central to elucidating whether P. marneffei is a saprotrophic fungus, and is the central hypothesis that we test here.

Surveys have shown that P. marneffei is endemic to India, Thailand, the Guangxi region of China, Vietnam, Taiwan and Hong Kong (Singh et al. 1999). Analysis of clinical isolates from these regions using MultiLocus Microsatellite Typing (MLMT) (Fisher et al. 2004a), has determined the population genetic structure of this fungus (Fisher et al. 2004b). Extensive phylogeographic structure was identified showing two deeply divided clades, corresponding to Eastern and Western southeast Asia. Within the Western clade, genetic differentiation was observed between spatially separated populations (Fisher et al. 2005). A consequence of this finding is that clinical isolates can be readily assigned to a geographic sourcepopulation. As these different genotypes may represent niche-adapted lineages, we included a representative isolate from both the 'East' and 'West' clades in our experiments, to compare their growth responses.

P. marneffei is closely related to other Penicillium species within the subgenus Biverticillium (Pitt 1979), and the genus Talaromyces has been identified as the closest relative to P. marneffei, which can exhibit sexual biverticilliate states (LoBuglio \& Taylor 1995). Of these, Talaromyces stipitatus is readily recovered from Thailand soils (Fisher, unpub. obs.). If niche-adaptation is important in generating the observed patterns of spatial genetic variation in P. marneffei, then it is likely that isolates from two different geographical regions, and representing both 'Western' and 'Eastern' clades, will show differing competitive abilities in soil. Further, if P. marneffei is a niche-specialist rather than an ubiquitous soil pathogen, then we can predict that it will perform poorly in competition with closely related fungal taxa.

This paper describes the detection of the pathogenic fungus $P$. marneffei from soils in Thailand. We then investigated the growth potential of the two P. marneffei genotypes in soil from northern Thailand, and measured the ability of these genotypes to compete directly with $\mathrm{T}$. stipitatus in soil microcosms. Relative growth of the fungi in our microcosms was measured using a rapid and reliable quantitative TaqMan probe-based quantitative PCR (qPCR) reaction (Haughland et al. 2004; Lotrario et al. 1995) following environmental DNA extraction. These experiments address an urgent need to better understand P. marneffei's survival and ecology in soil as infection is an important cause of morbidity and mortality in HIV patients living or travelling in Southeast Asia.

\section{Materials and methods}

\section{DNA sampling and extraction from soil}

Soil samples were collected over 3 years from locations where cases of penicilliosis had occurred and from a range of ecotypes in Northern Thailand within a circle of radius $60 \mathrm{~km}$, centered on Chiang Mai (Iat. $17.885^{\circ}$, Ion. 98.986 ). Environments sampled included the environs of eight patients houses who had fallen ill with penicilliosis marneffei, different forest and grassland environments, and sites associated with animals, such as bat-caves and an elephant camp. From each site, $20 \mathrm{~g}$ of soil was taken from the 'A' subsurface soil horizon to a maximum depth of $5 \mathrm{~cm}$. Total DNA was extracted from the soil samples using a modified flotation method (Larsh et al. 1953; Vanittanakom et al. 1995). Briefly, $30 \mathrm{ml}$ of phosphate buffered saline (PBS) solution was added to $15 \mathrm{~g}$ soil in a $50 \mathrm{ml}$ tube (Nunc $\mathrm{GmbH}$ \& Co. KG, Wiesbaden, Germany). The tubes were agitated by vortexing for $10 \mathrm{~min}$. The soil solution was then left to settle for $20 \mathrm{~min}$ at room temperature. As much supernatant as possible (approx $25 \mathrm{ml}$ ) was transferred with care to a sterile $50 \mathrm{ml}$ tube and topped up to $30 \mathrm{ml}$ with PBS solution. These soil extracts were centrifuged at $2400 \mathrm{~g}$ at $4{ }^{\circ} \mathrm{C}$ for $10 \mathrm{~min}$ and the supernatant was discarded. The pellet was suspended in $250 \mu$ l of sterile, distilled water and introduced into a Bead Solution tube (UltraClean ${ }^{\mathrm{TM}}$ Soil DNA Isolation Kit, Mo Bio Laboratories, Inc., Carlsbad, USA). Samples were bead-beaten (MiniBeadBeater-8, Biospec Products, Bartlesville, USA) for $2 \mathrm{~min}$ in the presence of $60 \mu \mathrm{l}$ of solution 1 and $200 \mu$ l of IRS solution (Biospec). The manufacturer's guidelines were then followed with the resulting DNA resuspended in $100 \mu \mathrm{l}$ sterile, distilled water. These soil DNA extracts were subsequently stored at $-20^{\circ} \mathrm{C}$. This protocol was followed for both environmental samples and soil microcosms.

\section{Quantitative environmental real-time PCR}

The qPCR assays were designed to target and amplify a $90 \mathrm{bp}$ portion of the $\beta$-tubulin locus, and were designed by alignment of $\beta$-tubulin sequences from Penicillium subgenus Biverticillium. DNA sequences for this region for 50 Penicillium and Talaromyces species were provided by the Centraalbureau voor Schimmelcultures (CBS). The sequences were aligned using ClustalW in MEGA version 3.0 (Kumar et al. 2004) and Primer Express 2.0 (Applied Biosystems) was used to design genera specific primers and species specific probes for P.marneffei and Talaromyces stipitatus. Real-time quantitative PCR 
utilised an internal probe which hybridised to a specific target sequence. The probe was labelled with two dyes, a fluorescent reporter at the $5^{\prime}$ end, a nonfluorescent quencher and minor groove binder at the $3^{\prime}$ end. The probes were designed adhering to the recommendations by Applied Biosystems so that there was less than $1^{\circ} \mathrm{C}$ difference between the two probe melting temperatures; each probe was positioned as closely as possible to the $3^{\prime}$ end of the TsF forward primer and the polymorphic site was placed in the central third of both probes.

We designed genera specific primer sequences as follows: TsF 5'CAAATCGGTGCTGCTTTCTG, TsR 5'CAGAGATGATTT GCCTGAAATAGTCA. The TaqMan probes that were used to discriminate the two species were: P. marneffei probe $5^{\prime}$ VIC-C TCTCAACAAACACATCAC-MGB $3^{\prime}$ and T. stipitatus probe $5^{\prime}$ 6FAM-TGCTTGGGTAACACTCA-MGB $3^{\prime}$.

The specificity of the MGB probes were tested by attempting to amplify the DNA extracted from several Penicillium and Talaromyces species: T. stipitatus (CBS375.48, USA); T. derxii (CBS412.89, Japan); P. verruculosum (CBS312.59, Japan); P. purpurpogenum (CBS286.36, Japan); P. funiculosum (CBS433.89, India) and P. rubrum (CBS184.27, USA). Amplifications were conducted in 96-well polypropylene plates on an ABI Prism 7300 (Applied Biosystems, Worthing, UK). Each $25 \mu$ l reaction contained $12.5 \mu \mathrm{l}$ of Taqman Universal PCR Mastermix (Applied Biosystems), $900 \mathrm{mM}$ of each primer (Eurogentec Ltd., Southampton, UK), $200 \mathrm{mM}$ of each minor groove binding (MGB) probe (Applied Biosystems), $5 \mu \mathrm{l}$ of a 1 in 10 dilution of the soil extract and $2.0 \mu \mathrm{l}$ of de-ionised water. The amplification conditions were $50^{\circ} \mathrm{C}$ for $2 \mathrm{~min}, 95^{\circ} \mathrm{C}$ for $10 \mathrm{~min}$ and then 50 cycles of $95^{\circ} \mathrm{C}$ for $15 \mathrm{sec}$ and $1 \mathrm{~min}$ at $60^{\circ} \mathrm{C}$. Each soil sample was tested in duplicate, only being considered positive if the cycle threshold $(\mathrm{Ct})$ value was under 45 cycles (Brancart et al. 2005). Standard curves based on Ct for a 10fold dilution series of P. marneffei genomic DNA $(n=3)$ were developed. The standard curves were obtained by plotting the $\mathrm{Ct}$ threshold versus the logarithm of the concentration of each 10 -fold dilution series. The dilution series ran over 4 orders of magnitude.

\section{Isolates used in microcosm competition experiments}

The following isolates were used: Western clade P. marneffei (CM; MMC47P11-5, Chiang Mai), Microsatellite Type (MT)=10; Eastern clade P. marneffei (TW; MY3370, Taiwan), MT=146 (http://pmarneffei.multilocus.net/); Talaromyces stipitatus (strain CBS375.48; Centraalbureau voor Schimmelcultures, Utrecht, The Netherlands). All isolates were grown on $2 \%$ malt extract agar (Smith 1994) at $28^{\circ} \mathrm{C}$ for 1 week. Spore dilutions were produced by scraping spores into $10 \mathrm{ml}$ of sterile distilled water. Subsequently, $20 \mu \mathrm{l}$ was added to a Neubauer chamber (Weber Scientific International Ltd, Teddington, England) and the number of spores counted under a microscope (Laborlex 12, Ernest Leitz Wetzlar, Germany). The average total number of $P$. marneffei spores $\mathrm{ml}^{-1}$ for each isolate was calculated. The concentrated spore solution was then diluted appropriately, with sterile distilled water, to produce a dilution series of $10^{8}, 10^{7}, 10^{6}, 10^{5}, 10^{4}, 10^{3}, 10^{2}, 10$ and 0 spores for each P. marneffei isolate and $10^{6}, 10^{5}, 10^{4}, 10^{3}, 10^{2}, 10$ and 0 spores for T. stipitatus.

\section{Competition experiment design}

Soil was collected from a single site outside the Maharaj Nakorn Chiang Mai Hospital, Chiang Mai (lat. 18.7899 ${ }^{\circ}$, lon. $\left.98.975^{\circ}\right)$. The soil was sieved and processed by serial autoclaving. Sterilisation was at $121^{\circ} \mathrm{C}$ for $1 \mathrm{~h}$, followed by cooling at room temperature for $24 \mathrm{~h}$, and then sterilised as before. This was repeated three times to ensure that any spores/enzymes stimulated by the first round of sterilisation would be destroyed in the second or third round (Bogan et al. 1996; Warcup, 2003).

Each microcosm comprised $15 \mathrm{~g}$ of sterile soil added to a Petri dish (Sterilin, Billingshurst, UK) and was inoculated with: $1 \mathrm{ml}$ of the appropriate P. marneffei spore dilution (focal species), $1 \mathrm{ml}$ of the appropriate T. stipitatus spore dilution (associate species) and the volume made up to $5 \mathrm{ml}$ of liquid with the addition of sterile distilled water. The spore numbers used for both strains were $0,10^{3}, 10^{4}$ and $10^{5}$, co-inoculated with T. stipitatus at $0,1,10^{2}, 10^{3}, 10^{4}$ and $10^{5}$ in all combinations, plus a control of no added spores of either species (Table 1). The competition experiments were performed in duplicate for each spore inoculum combination. The microcosms were all incubated at $28^{\circ} \mathrm{C}$ in the dark for $14 \mathrm{~d}$. As a non-sterile control, the same numbers of P. marneffei described above were inoculated in duplicate into air-dried, but non-sterile, soil to determine the ability of P. marneffei to survive 'naturally' in a soil microcosm that is complete with its normal complement of microbes.

\section{Statistical analyses}

We determined whether the increasing addition of $\mathrm{T}$. stipitatus spores had either a negative or positive impact upon: (1) $P$. marneffei survival; and (2) final DNA concentration at the end of the experiment. To aid data interpretation the cycle threshold (DNA detection) values were transformed into spore equivalents. As observation that the amplification reactions in this study sometimes did not exhibit perfect exponential amplification due to the presence of inhibitors from the soil extracts, detection rates (Ct value, Table 1) from a spore dilution series amplified in the presence of soil $(t=0 \mathrm{~d})$ were used to construct a standard curve plot in order to estimate fungal biomass increase as 'spore equivalents'.

Statistical tests were carried out using ANOVA in S-PLUS (Lucent Technologies 2003). Due to non-normal distribution of the experimental residuals, an inverse of the cube transformation was used to normalise the distribution of data.

\begin{tabular}{|c|c|c|}
\hline $\begin{array}{l}\text { Spores } \\
\text { inoculated } \\
(0 \mathrm{~d})\end{array}$ & $\begin{array}{c}\text { P. marneffei CM, } \\
\text { spore equivalents } \\
(14 \mathrm{~d})\end{array}$ & $\begin{array}{c}\text { P. marneffei TW, } \\
\text { spore equivalents } \\
\text { (14 d) }\end{array}$ \\
\hline $10^{3}$ & $9.8 \times 10^{4} \pm 18000$ & $9.7 \times 10^{4} \pm 11000$ \\
\hline $10^{4}$ & $5.7 \times 10^{5} \pm 1000$ & $2.7 \times 10^{5} \pm 27000$ \\
\hline $10^{5}$ & $8 \times 10^{5} \pm 6600$ & $3.8 \times 10^{5} \pm 28000$ \\
\hline
\end{tabular}


However, actual results using transformed and non-transformed data did not differ substantially and, in particular, there was little difference in the final levels of significance.

\section{Results}

Real-time primer and TaqMan ${ }^{\circledR}$ probe design and specificity

Generic primers TsF/R, were chosen from the alignment of $\beta$ tubulin sequences from biverticilliate and terverticilliate Penicillium and Talaromyces species. Using this primer pair to amplify genomic DNA from 9 isolates of Penicillium and Talaromyces, resulted in PCR products that were in all cases c. 90 base pairs consistent with the in silico predictions of 90 base pairs for P. marneffei and 91 base pairs for T. stipitatus. This size PCR product allowed species specific probes to be developed - P. marneffei (Pm_Btub) and T. stipitatus (Ts_Btub); there was no detection of the seven other Penicillium and Talaromyces species. A threshold cycle, Ct, value of zero was determined for the 5 isolates ( $n=9$ per isolate). Only P. marneffei and T. stipitatus were amplified with Ct values of 18.9 and 20.3, respectively.

Extraction, then amplification of DNA from soil samples that had been spiked with P. marneffei spores showed that DNA was successfully detected down to 10 spores $\mathrm{ml}^{-1}$ (Fig 1).

\section{Environmental detection of P. marneffei}

Of 137 sites tested for P. marneffei, seven were positive (5.1\%). Of the positive sites, one of eight patient's homes - a sample from the base of a tree - was associated with a positive qPCR test. Correct detection of P. marneffei was confirmed in some cases by sequencing the amplification products for the intragenic (ITS) spacer region. In all cases, there was $100 \%$ homology with published P. marneffei ITS sequences (data not shown). The positive soil samples were from: (1) inside a cave populated by bats; (2) an elephant camp; (3) outside a patient's house; and (4) near a Buddhist Temple. Positive samples were recovered from Elephant-camp soils for each

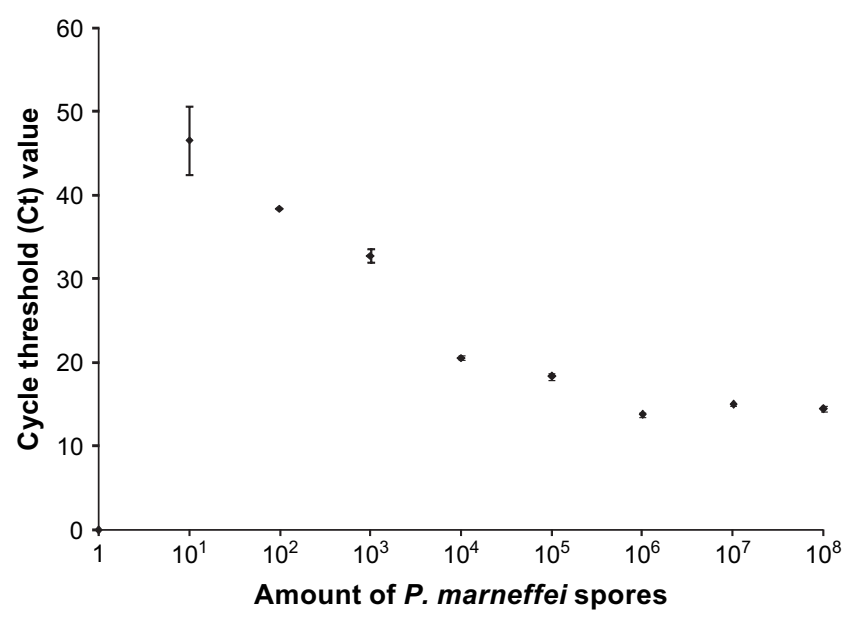

Fig 1 - Detection of P. marneffei from a dilution series of soils spiked with $10^{8}-1$ spores. of the three sampling years (Fig 2; www.spatialepidemiology.net/pmarneffei).

\section{P. marneffei survival in sterile and non-sterile soil}

Neither P. marneffei isolate could be detected, after $14 \mathrm{~d}$, by real-time PCR ( $n=8$, for each isolate) when spores were inoculated into non-sterile soil, irrespective of the initial spore concentration (Fig 3). However, both P. marneffei isolates, CM (Chiang Mai) and TW (Taiwan), were detected in the sterile soil microcosms (Fig 3) after the same time. The lowest spore inoculum, $10^{3}$ spores, was detected for both CM and TW isolates with similar $\mathrm{Ct}$ values (Ct38.99 \pm 1.82 for $\mathrm{CM}$ and $\mathrm{Ct} 39.80 \pm 1.14$ for TW) following $14 \mathrm{~d}$ incubation (Table 1).

Growth of P. marneffei CM and TW isolates in sterile soil with no added T. stipitatus

Increasing spore inoculum resulted in increased DNA detection (Fig 1). After $14 \mathrm{~d}$ of incubation, $9.8 \times 10^{4}( \pm 18000)$ spore equivalents were detected where $10^{3}$ spores of $\mathrm{CM}$ had been inoculated, increasing to $8.2 \times 10^{5}( \pm 6600)$ spore equivalents for $10^{5}$ spores of CM inoculated (Tables 2 and 3). Similarly, after $14 \mathrm{~d}, 9.7 \times 10^{4}( \pm 11000)$ spore equivalents were detected in microcosms inoculated with $10^{3} \mathrm{P}$. marneffei TW spores. Microcosms inoculated with $10^{5}$ spores of TW showed increased biomass equal to the detection of $3.8 \times 10^{5}( \pm 28000)$ spore equivalents. There was no significant difference $(P>$ 0.05 ) in the biomass produced by $P$. marneffei $C M$ and TW at equivalent spore inoculum concentrations (Fig 4A).

\section{P. marneffei competitive response to increasing \\ T. stipitatus spore concentrations}

Real-time PCR detected both P. marneffei strains in all of the DNA extracts from the sterile microcosms to which they were added. The initial P. marneffei spore inoculum

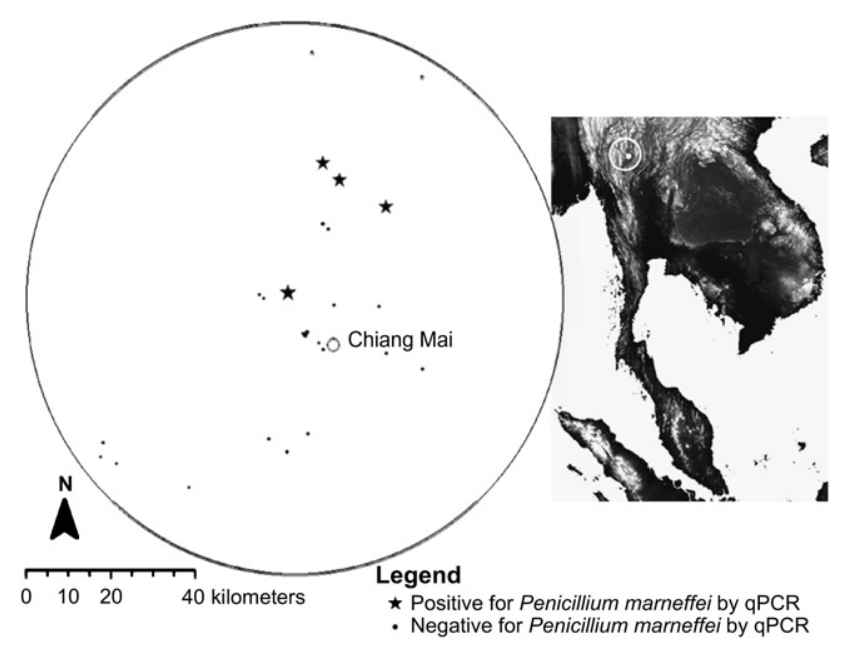

Fig 2 - Map of the locations of sample sites around Chiang Mai, Thailand. $\star$ Site positive for P. marneffei by $\mathrm{qPCR}$. $\bullet$ Site negative for P. marneffei by qPCR. For dynamic colour map see: http://www.spatialepidemiology.net/pmarneffei/env/. 


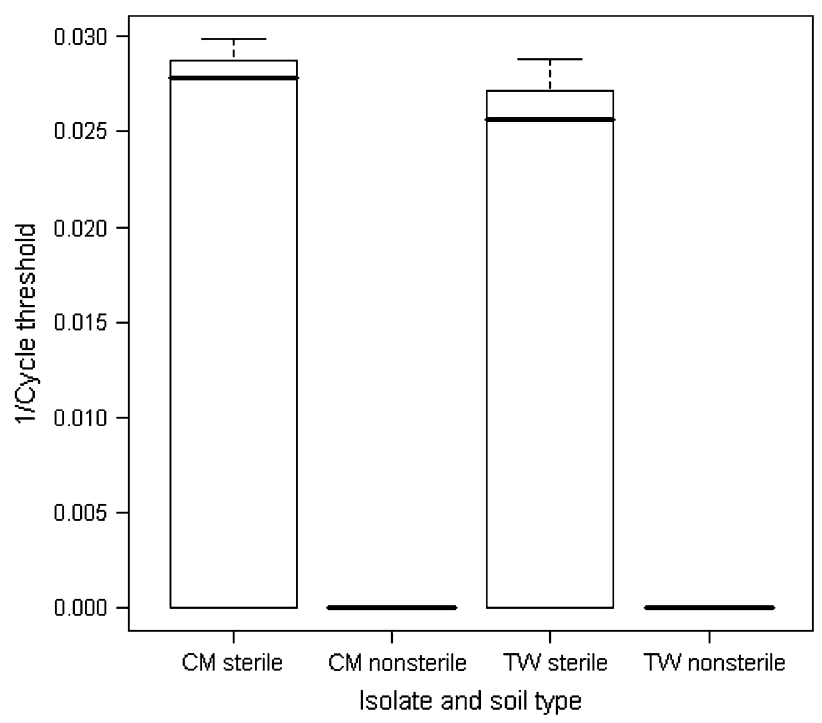

Fig 3 - Detection of the P. marneffei isolates (CM - from Chiang Mai and TW - from Taiwan) $14 \mathrm{~d}$ after inoculation into sterile and non-sterile soils.

significantly effected the level of P. marneffei detection (CM $\left.F_{1,112}=37.7, P=1.27 \times 10^{-8} ; \mathrm{TW} \mathrm{F}_{1,77}=1.3, \mathrm{P}=1.8 \times 10^{-5}\right)$. The impact of $T$. stipitatus on the growth of the two $P$. marneffei isolates in soil, as estimated using ANOVA, was non-significant $\left(\mathrm{CM} \mathrm{F}_{1,112}=37.7, \mathrm{P}=0.075 ; \mathrm{TW} \mathrm{F}_{1,77}=1.3, \mathrm{P}=0.26\right)$.

Growth of P. marneffei TW was reduced by $11 \%$, in the treatment with $10^{5} \mathrm{~T}$. stipitatus spores added compared to microcosms with no added spores, but this was not significant $(P>0.05)$. Both $P$. marneffei isolates persisted and increased in biomass in all microcosms.

\section{T. stipitatus response to increasing spore concentrations of P. marneffei}

ANOVA showed that initial spore concentration was the most significant factor influencing $\mathrm{Ct}$ end point biomass (CM, $\left.F_{1,74}=79.3, P=2.53 \times 10^{-13} ; \mathrm{TW}, F_{1,81}=29.6, P=5.4 \times 10^{-7}\right)$. When co-inoculated with P. marneffei CM, T. stipitatus was not detected in six of the treatments (Fig 4B). Fewer than 10 to 100 spore equivalents were present after $14 \mathrm{~d}$ of competition with P. marneffei CM.

Table 3-Mean cycle threshold value for $T$. stipitatus at the five spore concentrations co-inoculated with the two isolates of $P$. marneffei

Number of

T. stipitatus spores inoculated

P. marneffei isolate (Chiang Mai, CM)

P. marneffei isolate

\begin{tabular}{lcr}
\hline $10^{1}$ & 0 & $39.4 \pm 1.04$ \\
$10^{2}$ & 0 & $39.9 \pm 1.46$ \\
$10^{3}$ & $40 \pm 0.76$ & $38.1 \pm 1.42$ \\
$10^{4}$ & $36.67 \pm 1.28$ & $33.27 \pm 1.42$ \\
$10^{5}$ & $32.70 \pm 1.22$ & $32.3 \pm 0.90$ \\
\hline
\end{tabular}

T. stipitatus was however detected in all of the microcosms into which it was inoculated with P. marneffei TW (Fig 3). There was a significant $(P \leq 0.05)$ decrease in the $C$ t value when over $10^{6}$ spores were added to the microcosms. Detection of T. stipitatus occurred earlier in the microcosms which were inoculated with $P$. marneffei TW isolate (Table $2 ; F_{1,155}=4.6, P=0.03$ ).

\section{Discussion}

Our results have shown that P. marneffei is clearly present in soils within Thailand, but as DNA was only detected in seven of 137 sampled environments, it was not possible to analyse specific associations between occurrence and environmental variables, or whether there was a non-random distribution of P. marneffei in the environment. However, it is worth noting that three of the seven sites are heavily used by animals (a bat cave, an elephant camp and a human habitation with a known history of P. marneffei infection), and in one site (the elephant camp) P. marneffei DNA was detected over several years. As P. marneffei is known to be associated with Bamboo rats, our observations are circumstantial evidence that the organism may be associated with a wider range of species than is currently known.

The molecular qPCR test that we have developed is shown here to be highly sensitive, detecting 10 P. marneffei spores $\mathrm{ml}^{-1}$ in spiked soil samples. However, in our environmental survey for the pathogen, we were not able to distinguish between live, viable P. marneffei conidia and inviable conidia. We attempted to culture the organism from PCRpositive sites, however, were not successful. The use of mRNA in reverse transcriptase PCRs is today enabling both the symptomatic and presymptomatic detection of viable

Table 2 - Cycle threshold (Ct) values and number of spores for the two isolates of P. marneffei (CM, TW) after 0 and $14 \mathrm{~d}$ in soil microcosms

\begin{tabular}{|c|c|c|c|c|c|}
\hline \multirow{2}{*}{$\begin{array}{l}\text { Number of spores } \\
\text { inoculated }\end{array}$} & \multirow{2}{*}{$\begin{array}{l}\text { P. marneffei at } 0 \mathrm{~d} \text { cycle } \\
\text { threshold value }\end{array}$} & \multicolumn{2}{|c|}{ P. marneffei at $14 \mathrm{~d}$} & \multicolumn{2}{|c|}{ P. marneffei at $14 \mathrm{~d}$} \\
\hline & & Ct value & Spores & Ct value & Spores \\
\hline $10^{3}$ & $\mathrm{a}$ & $38.99 \pm 1.82$ & $9.8 \times 10^{4} \pm 18000$ & $39.80 \pm 1.14$ & $9.7 \times 10^{4} \pm 11000$ \\
\hline $10^{4}$ & a & $35.70 \pm 1.04$ & $5.7 \times 10^{5} \pm 10000$ & $37.63 \pm 2.67$ & $2.7 \times 10^{5} \pm 27000$ \\
\hline $10^{5}$ & $38.46 \pm 0.86$ & $34.06 \pm 0.66$ & $8.2 \times 10^{5} \pm 6600$ & $36.83 \pm 2.84$ & $3.8 \times 10^{5} \pm 28000$ \\
\hline $10^{6}$ & $32.9 \pm 0.22$ & b & - & $\mathrm{b}$ & - \\
\hline $10^{7}$ & $28.34 \pm 0.51$ & $\mathrm{~b}$ & - & b & - \\
\hline
\end{tabular}



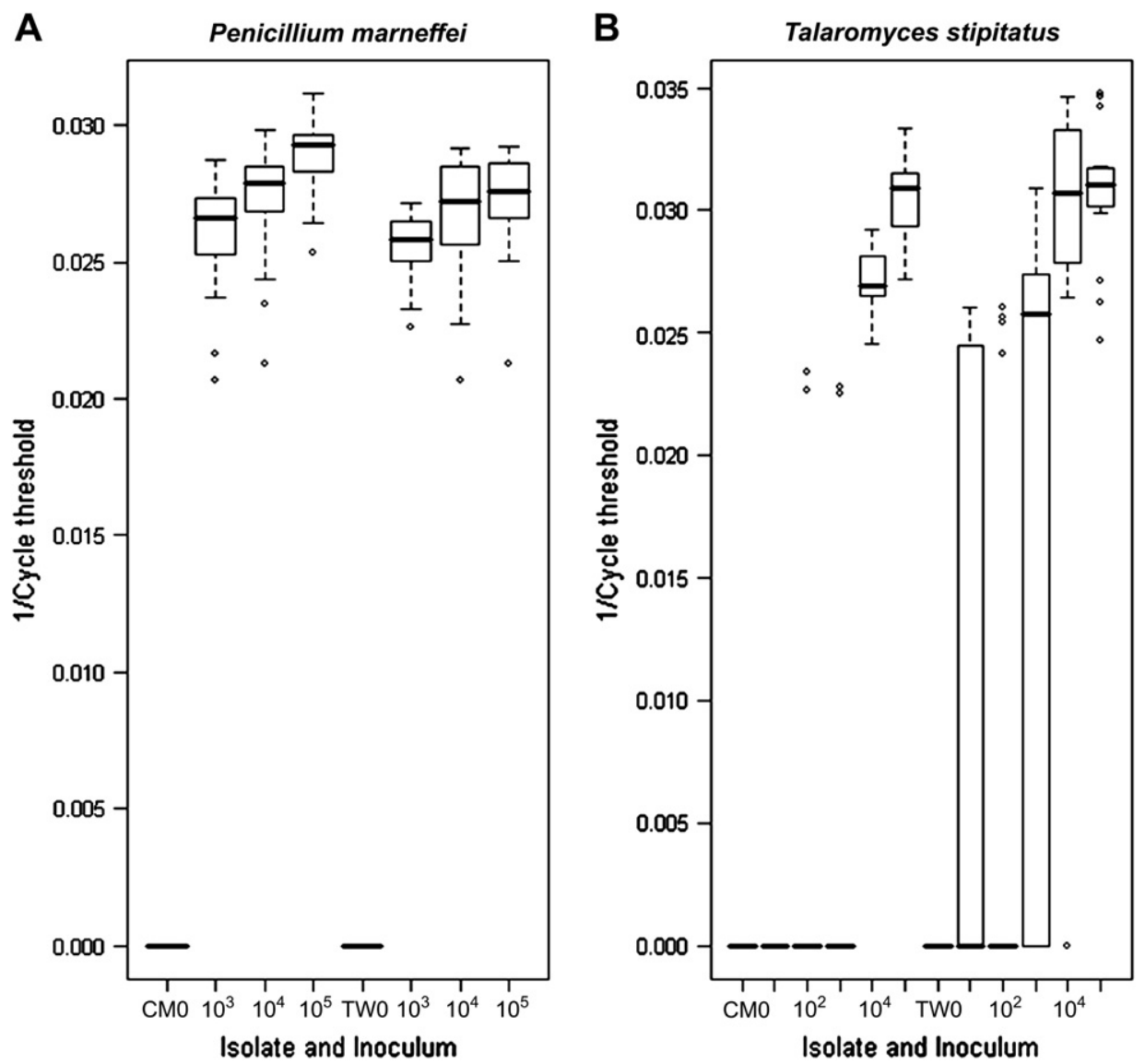

Fig 4 - Quantification of A. Penicillium marneffei and B. Talaromyces stipitatus when grown in direct competition in sterile soils after 14 d. (A). P. marneffei grown in competition with 100000 T. stipitatus spores. (B). T. stipitatus grown in competition with 100000 P. marneffei spores. $\mathrm{CM}=$ Chiang Mai P. marneffei and TW $=$ Taiwan $P$. marneffei isolate.

fungal pathogens in plants (Gough \& Lee 1985; McMaugh \& Lyon 2003) and our future experiments aim to investigate cell viability by using $P$. marneffei-specific probes to the mRNA of a constitutively-expressed gene such as beta tubulin (Yan \& Liou 2006).

P. marneffei is not very competitive with soil microflora and microfauna in non-sterile microcosms and DNA was below the limits of detection by $14 \mathrm{~d}$ after inoculation. The only other studies to date examining the efficacy of detecting P. marneffei in seeded non-sterile and sterile soil used traditional culturing methods (Vanittanakom et al. 1995; Joshi et al., 2003). In Vanittanakom et al.'s study the recovery rate from 100 P. marneffei spores in non-sterile soil was $6 \%$ compared to $84.3 \%$ from sterile soil, mirroring our finding that P. marneffei does not flourish naturally in soil. As yet, no other published study has attempted to monitor P. marneffei in soil using molecular means. However, a 2-fold reduction in fungal detection efficiency using real-time PCR was recorded when directly quantifying Glomus intraradices from non-sterile soil (Filion et al. 2003). Our study suggests that not only does $P$. marneffei survival in non-sterile soil suffer, but so does qPCR efficiency. There are several reasons for the differences between sterile and non-sterile soil. Autoclaving alters the chemical composition of soil (Salonius et al. 1967), removes competition from soil organisms, makes available nutrient resources and slightly raises the $\mathrm{pH}$ (Lotrario et al. 1995). In natural soils mycostatic and mycolytic factors commonly occur and are known to adversely affect the development of some human pathogenic fungi (McDonough 1963). Such factors may be negatively affected by the sterilization process. Further, in non-sterile soil microcosms, intact non-target soil micro-flora present may negatively influence real-time PCR amplification and in turn the levels of fungi detected (Filion et al. 2003).

In the present study, growth of P. marneffei was readily detected in the sterile microcosms. Since the detectable level of inoculated soil at $0 \mathrm{~d}$ was $10^{5}$ spores, but after $14 \mathrm{~d}$ detection was possible in the microcosms to which $10^{3}$ spores were added, DNA extraction has increased 100-fold. This presumably reflects an increase in fungal biomass over the $14 \mathrm{~d}$. P. marneffei has also been isolated from inoculated sterile Thai soil in two other studies (Vanittanakom et al. 1995).

The model microcosm experiments demonstrated that, when in direct competition with a closely related Trichocomaceae species, P. marneffei establishment and growth was not significantly affected. T. stipitatus, when added to the study system, did not elicit a negative competitive response from P. marneffei CM, which was not competitively excluded from any of the microcosms. Although not statistically significant, it was noted that P. marneffei TW did not increase in biomass as consistently when in the presence of a high concentration 
of T. stipitatus spores. This is preliminary evidence that P. marneffei $\mathrm{CM}$ exhibited higher relative fitness in the Chiang Mai soil microcosms when compared to P. marneffei TW, although the effect was not statistically significant under our current levels of experimental replication. The data suggest that $T$. stipitatus was able to grow more effectively when in competition with P. marneffei TW than with P. marneffei CM.

However, it should be noted that the many studies on DNA extraction methods have highlighted the difficulty of consistent extraction efficiency and PCR processing, especially in relation to extractions from soil (Filion et al. 2003; MartinLaurent et al. 2001; Miller et al. 1999; Yeates et al. 1999). Extraction efficiency varies due to factors such as adsorption to soil colloids (Frostegard et al. 1999) and the co-extraction of humic substances (Zhou et al. 1996). Whilst further optimisation of our experimental setup is required, such methodological variables should not impact on the findings of our study as all fungal microcosms were processed in an identical manner.

As both fungal species used in this study are closely related, it is likely that they share similar life strategies. Typically, Penicillium and Talaromyces include $r$-selected characteristics required for successful primary resource capture, namely good dispersal, rapid spore germination, rapid mycelial extension, and the ability to utilise organic substances available in previously uncolonised resources (Cooke \& Rayner 1984). While, there was some negative interaction at low T. stipitatus spore concentrations, there were no dramatic competitive responses by either fungus. This may be explained by either: (1) the two fungi occupying different niches; or (2) sharing similar functional capabilities and initial resources important for colony establishment, but with their functional capabilities diversifying and separating with colony maturation.

P. marneffei exhibits highly structured populations (Fisher et al. 2005), and one mechanism by which this structure could have evolved is local adaptation to heterogeneous environments. This apparently, clonal asexual fungus co-colonised the soil microcosms successfully and flourished in the presence of a closely related competitor. We observed a significant effect of altering soil type on the growth of P. marneffei, however we were not able to do the reciprocal experiment using a 'typical' Taiwanese soil. Until this experiment is repeated with greater numbers of isolates from a larger number of regions, it is not possible to say whether our results have a general significance. However, our data raise interesting questions, and potential answers, as to why there are such deep divisions in the population genetic structure of P. marneffei.

Our finding that P. marneffei is unable to grow within an unsterilised environment suggests that its niche in nature may be more specific than that which we used here. The dispersal and growth of fungi strongly depends on environmental factors; among these soil type, rainfall, humidity are all expected to control germination and growth (Frostegard et al. 1999). Our finding that P. marneffei cannot survive in non-sterile microcosms is not a refutation of the soil microenvironment as a reservoir of the fungus. Rather, these data show that we need to consider wider ranges of environmental variables and spatial scales when investigating the metapopulation structure of these pathogenic fungi in nature.
In conclusion, this study has shown that P. marneffei is present in soil in endemic regions in Thailand, and that soil microcosms provide a tool by which the dynamics of its establishment, growth and resource partition can be monitored by quantitative molecular methods. Further studies on the ability of P. marneffei to function in more complex fungal communities, and in different environmental backgrounds, will provide a better understanding of the interplay between fitness, function and the population genetics on the metapopulation dynamics of pathogenic fungi.

\section{Acknowledgements}

The Taiwanese isolate of P. marneffei was supplied by PR Hsueh, $\beta$-tubulin alignments were provided by S de Hoog, Centraal Bureau voor Schimmelcultures, the Netherlands, and the research was funded by a Wellcome Trust Biodiversity grant to MC Fisher.

\section{R E F E R E N C E S}

Ajello LPA, Sukroongreung S, Nilakul CH, Tantimavanic S, 1995. Occurrence of Penicillium marneffei infections among wild bamboo rats in Thailand. Mycopathologia 131: 1-8.

Bogan B, Schoenike B, Lamar R, Cullen D, 1996. Manganese peroxidase mRNA and enzyme activity levels during bioremediation of polycyclic aromatic hydrocarboncontaminated soil with Phanerochaete chrysosporium. Applied and Environmental Microbiology 62: 2381-2386.

Brancart F, Rodriguez-Villalobos H, Fonteyne P-A, Peres-Bota D, Liesnard C, 2005. Quantitative TaqMan PCR for detection of Pneumocystis jiroveci. Journal of Microbiological Methods 61: 381-387.

Capponi M, Sureau P, Segretain G, 1956. Penicillose de Rhizomys sinensis. Bulletin of the Exotic Pathology Society 49: 418-421.

Chariyalertsak S, Vanittanakom P, Nelson KE, Sirisanthana T, Vanittanakom N, 1996. Rhizomys sumatrensis and Cannomys badius, new natural animal hosts of Penicillium marneffei. Journal of Medical and Veterinary Mycology 34: 105-110.

Chariyalertsak S, Sirisanthana T, Supparatpinyo K, Praparattanapan J, Nelson KE, 1997. Case-control study of risk factors for Penicillium marneffei infection in human immunodeficiency virus-infected patients in northern Thailand. Clinical Infectious Diseases 24: 1080-1086.

Cooke RC, Rayner ADM, 1984. The Ecology of Saprotrophic Fungi. Longman, London.

Deng ZL, Yun M, Ajello L, 1986. Human Penicilliosis marneffei and its relation to the bamboo rat (Rhizomys pruinosus). Journal of Medical and Veterinary Mycology 24: 383-389.

Filion M, St-Arnaud M, Jabaji-Hare SH, 2003. Direct quantification of fungal DNA from soil substrate using real-time PCR. Journal of Microbiological Methods 53: 67-76.

Fisher MC, Aanensen D, de Hoog S, Vanittanakom N, 2004a. Multilocus microsatellite typing system for Penicillium marneffei reveals spatially structured populations. Journal of Clinical Microbiology 42: 5065-5069.

Fisher MC, de Hoog GS, Vanittanakom N, 2004b. A highly discriminatory Multilocus Microsatellite Typing System (MLMT) for Penicillium marneffei. Molecular Ecology Notes 5: 231-234.

Fisher MC, Hanage WP, de Hoog S, Johnson E, Smith MD, White NJ, Vanittanakom N, 2005. Low effective dispersal of 
asexual genotypes in heterogeneous landscapes by the endemic pathogen Penicillium marneffei. PLoS Pathogens 1: 159-165.

Frostegard A, Courtois S, Ramisse V, Clerc S, Bernillon D, Le Gall F, Jeannin P, Nesme X, Simonet P, 1999. Quantification of bias related to the extraction of DNA directly from soils. Applied and Environmental Microbiology 65: 5409-5420.

Gough FJ, Lee TS, 1985. Moisture effects on the discharge and survival of conidia of Septoria tritici. Phytopathology 75: 180-182.

Grishkan I, Nevo E, Wasser SP, Beharav A, 2003. Adaptive spatiotemporal distribution of soil microfungi in 'Evolution Canyon' II, Lower Nahal Keziv, western Upper Galilee, Israel. Biological Journal of the Linnean Society 78: 527-539.

Haugland RA, Varma M, Wymer LJ, Vesper SJ, 2004. Quantitative PCR analysis of selected Aspergillus, Penicillium and Paecilomyces species. Systematic and Applied Microbiology 27: 198-210.

Johansson M, 2001. Composition of the saprotrophic fungi in Calluna heathland soil and the influence of ammonium nitrate application. Water, Air and Soil Pollution: Focus 1: 231-239.

Joshi A, Gugnani HC, Vijayan VK, 2003. Survival of Penicillium marneffei in sterile and unsterile soil. Journal of Medical Mycology 13: 211-212.

Kjøller A, Struwe S, 1982. Microfungi in ecosystems: fungal occurrence and activity in litter and soil. Oikos 39: 391-422.

Kumar S, Tamura K, Nei M, 2004. MEGA3: integrated software for molecular evolutionary genetics analysis and sequence alignment. Briefings in Bioinformatics 5: 150-163.

Larsh H, Hinton A, Furcolow M, 1953. Laborotory studies of Histoplasma capsulatum III. Efficiency of the flotation method in isolation of Histoplasma capsulatum from soil. The Journal of Laboratory and Clinical Medicine 41: 478-485.

LoBuglio K, Taylor J, 1995. Phylogeny and PCR identification of the human pathogenic fungus Penicillium marneffei. Journal of Clinical Microbiology 33: 85-89.

Lotrario JB, Stuart BJ, Lam T, Arands RR, O'Connor OA, Kosson DS, 1995. Effects of sterilization methods on the physical characteristics of soil: implications for sorption isotherm analyses. Bulletin of Environmental Contamination and Toxicology 54: 668-675.

Lucent Technologies. I. S plus. 2003.

Martin-Laurent F, Philippot L, Hallet S, Chaussod R, Germon JC, Soulas G, Catroux G, 2001. DNA Extraction from soils: old bias for new microbial diversity analysis methods. Applied and Environmental Microbiology 67: 2354-2359.
McDonough E, 1963. Effects of natural soils on Blastomyces dematitidis, Histoplasma capsulatum and Allescheria boydii. American Journal of Hygiene 77: 66-72.

McMaugh SJ, Lyon BR, 2003. Real-time quantitative RT-PCR assay of gene expression in plant roots during fungal pathogenesis. Biotechniques 34: 982-986.

Miller DN, Bryant JE, Madsen EL, Ghiorse WC, 1999. Evaluation and optimization of DNA extraction and purification procedures for soil and sediment samples. Applied and Environmental Microbiology 65: 4715-4724.

Paul EA, Clarke FE, 1970. The microflora of grassland. Advances in Agronomy 22: 375-435.

Pitt JI, 1979. The Genus Penicillium and its Teleomorphic States Eupenicillium and Talaromyces. Academic Press, Inc., New York.

Pitt JI, 1988. Laboratory Guide to Common Penicillium Species. Academic Press, London, New York, Toronto, Sydney, SanFrancisco.

Salonius PO, Robinson JB, Chase FE, 1967. A comparison of autclaved and gamma-irradiated soils as medi for microbial colonisation experiments. Plant and Soil 27: 239-248.

Singh PN, Ranjana K, Singh YI, Singh KP, Sharma SS, Kulachandra M, Nabakumar Y, Chakrabarti A, Padhye AA, Kaufman L, Ajello L, 1999. Indigenous disseminated Penicillium marneffei infection in the state of Manipur, India: report of four autochthonous cases. Journal of Clinical Microbiology 37: 26992702.

Smith DO, 1994. The Preservation and Maintenance of Living Fungi. In: IMI Technical Handbook, second ed, vol 2. CAB International, Wallingford.

Supparatpinyo KC, Khamwan V, Baosoung KE, Nelson KE, Sirisanthana T, 1994. Disseminated Penicillium marneffei infection in southeast Asia. Lancet 344: 110-113.

Vanittanakom N, Mekaprateep M, Sriburee P, Vanittanakom P, Khanjanasthiti P, 1995. Efficiency of the flotation method in the isolation of Penicillium marneffei from seeded soil. Journal of Medical and Veterinary Mycology 33: 271-273.

Warcup JH, 2003. Soil steaming: a selective method for the isolation of ascomycetes from soil. Transactions of the British Mycological Society 34: 515-518.

Yan HZ, Liou RF, 2006. Selection of internal control genes for realtime quantitative RT-PCR assays in the oomycete plant pathogen Phytophthora parasitica. Fungal Genetics and Biology 43: 430-438.

Yeates CG, Davison AD, Altavilla N, Veal DA, 1999. Methods for microbial DNA extraction from soil for PCR amplification. Biological Procedures Online 1: 40-47.

Zhou J, Bruns M, Tiedje J, 1996. DNA recovery from soils of diverse composition. Applied and Environmental Microbiology 62: 316-322. 